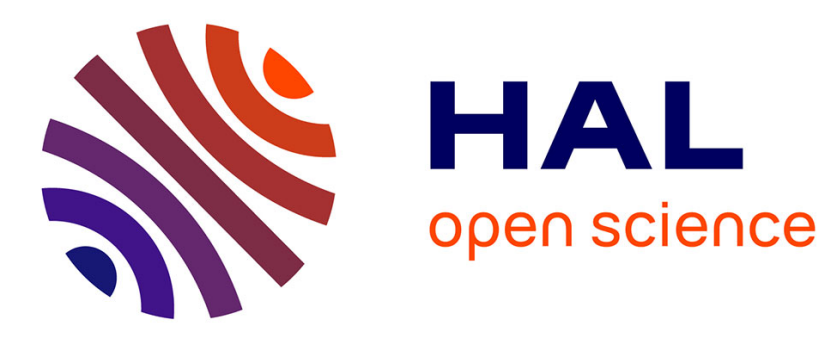

\title{
A Goal-Based Framework For Supporting Medical Assistance: The Case of Chronic Diseases
}

Milene Santos Teixeira, Célia da Costa Pereira, Mauro Dragoni

\section{To cite this version:}

Milene Santos Teixeira, Célia da Costa Pereira, Mauro Dragoni. A Goal-Based Framework For Supporting Medical Assistance: The Case of Chronic Diseases. PRIMA 2020:The 23rd International Conference on Principles and Practice of Multi-Agent Systems, Nov 2020, Nagoya (virtual), Japan. 10.1007/978-3-030-69322-0_18. hal-03199543

\section{HAL Id: hal-03199543 https://hal.science/hal-03199543}

Submitted on 15 Apr 2021

HAL is a multi-disciplinary open access archive for the deposit and dissemination of scientific research documents, whether they are published or not. The documents may come from teaching and research institutions in France or abroad, or from public or private research centers.
L'archive ouverte pluridisciplinaire HAL, est destinée au dépôt et à la diffusion de documents scientifiques de niveau recherche, publiés ou non, émanant des établissements d'enseignement et de recherche français ou étrangers, des laboratoires publics ou privés. 


\title{
A Goal-Based Framework For Supporting Medical Assistance: The Case of Chronic Diseases
}

\author{
Milene Santos Teixeira ${ }^{1}$, Célia da Costa Pereira ${ }^{2}$, and Mauro Dragoni ${ }^{3}$ \\ 1 University of Trento, Italy m. santosteixeira@unitn. it \\ ${ }^{2}$ Université de Nice Sophia Antipolis, France celia.pereira@unice.fr \\ ${ }^{3}$ Fondazione Bruno Kessler, Italy dragoni a fbk . eu
}

\begin{abstract}
Chronic diseases cause many deaths worldwide each year. The most common action to manage this type of disease is to conduct expensive pathological tests whose goal is to assess risks and identify early complications to patients health or to prevent the patient from developing other diseases. The choice of which action/test to perform is therefore important. There are in the literature some guideline-based decision-support systems which help to assist practitioners to choose the appropriate therapeutic action for their patients. The idea of such systems is to structure a guideline as a set of choices to be made by the clinician. However, such a set of actions/tests/decisions can change according to new information obtained concerning the patient state (direct answers from the patient, blood test results, etc.). Because the time factor may be crucial and because executing tests is expensive, it is then important for the practitioners to ask for or to quickly obtain useful information helping take the good decision in order to achieve the goals of excluding the risks as soon as possible, which means with a minor quantity of information/tests required. In this paper, we address the challenges introduced above by proposing an agent-based framework that supports the development of an intelligent goal-driven agent to help practitioners in choosing the most useful action to perform (question to ask a patient, test, etc.) in the case of a chronic disease. The framework supports the selection of the next dialogue action by measuring the usefulness, with respect to a goal, of a piece of information to be obtained. We introduce our framework through the use of a running example, showing how an agent can drive the interaction based on both its background knowledge and the new information it acquires. Experiments performed concerning two chronic diseases, namely asthma and type-2 diabetes, validate our approach. Finally, we discuss further possible scenarios where our framework can be applied in different ways.
\end{abstract}

\section{Introduction and Motivations}

Chronic diseases cause many deaths worldwide each year [1]. Many works in the literature propose solutions to help decreasing this number. Most of them propose solutions to assist health care practitioners, thus helping them to take good decisions according to the patient health conditions [2]3] and other works aim at assisting the patients managing themselves - Self-monitoring of health [4]5|6778|9]. However, as it has been underlined in [10], medical assistance combine medical actions performed by health care professionals who observe signs and symptoms and decide on questions, interventions, prescriptions or tests to treat the health problem of a particular patient. A recent study 
by [11] proposes a review of monitoring strategies in current United kingdom guidelines for patients with type 2 diabetes, chronic kidney disease, and hypertension. This study highlighted the uncertainties in the guidelines and the need for further research. Manning, who studied several publishers of clinical guideline resources for practitioners [12], suggested that primary care Information Technology providers should develop Artificial Intelligence (AI) engines that would interact with the data acquired by the physician during the consultation (free text, reading codes, etc.) and to link such data to the National Institute for Health and Care Excellence guidelines on specific conditions (background knowledge).

The agent-based framework we are proposing here which supports the development of an intelligent goal-driven agent to help practitioners in choosing the most useful action to perform in the case of a chronic disease, is in this line. Indeed, because the most common action to manage chronic diseases is to conduct expensive pathological tests whose goal is to assess risks and identify early complications to patients' health or to prevent the patient from developing other diseases, the choice of which action/test to perform is therefore important: the time factor may be crucial and executing tests is expensive. It is then important for the practitioners to ask for or to quickly obtain useful information helping take the good decision in order to achieve the goals of excluding the risks as soon as possible, which means with a minor quantity of information/tests required. This is the main aim of our framework: to be used by practitioners during a consultation and support her in the process of selecting the next dialogue action by measuring the usefulness, with respect to a risky situation (goal), of a piece of information to be obtained. Here, as dialogue, we mean the whole interaction process between an agent and the environment (practitioners, sensors, services, etc.) and not limited to a conversation between the agent and the human actor.

We start from the framework proposed in [13] whose aim is to characterize how useful a piece of information is for a cognitive agent which has some beliefs and goals. Here, we adapt and extend it by introducing several elements, enabling its application into a complex real-world problem: helping managing chronic diseases in the healthcare domain.

We consider different types of information which are stored by categories (set of slots): (i) $S_{a}$ : information acquired directly by asking the patient, (ii) $S_{b}$ : information acquired directly through sensors, (iii) $S_{c}$ : information acquired from the patient's electronic file, (iv) $S_{d}$ : information acquired from the history of data provided by the patient, (v) $S_{e}$ : information acquired through external services. Depending on the domain, each set of slots can be instantiated with the information needed. For example, in the case of diabetes, type $S_{a}$ slots corresponds to the categories of foods consumed, type $S_{b}$ slots to the measured blood sugar levels, type $S_{d}$ slots to what the patient ate during the week. In the case of asthma, those of type $S_{a}$ correspond to the symptoms that the patient reports, those of type $S_{b}$ to the breathing measured by a device, those of type $S_{e}$ to the data of the pollen controllers. In a real-time monitoring system for crisis prevention and/or compliance with treatment plans, all goals are coded starting from the guidelines and the system must prevent the patient from going to put himself in situations at risk - the different goals are to save the patient from the risky situations identified in the guidelines. Our work is in line with the framework used in [3]. However, in addition to what is proposed in that work, which is based on the measurement of all values (obtained from sensors), our framework give us also the possibility to (i) choose the 
most useful measurement to do, (ii) obtain information from the patient and use it when making a decisions, and to (iii) use background knowledge.

The overall result is the generation of a multi-turn interactions that uses as few questions as possible to retrieve the usefull information, seeking for a fast, but also cautious advises to the patient. As we can see from the experiment results, the approach is domain-configurable, that is, it can be applied to different domains within healthcare by modeling the domain knowledge properly.

\section{Preliminaries}

In this section, we will present some definitions composing our framework.

Definition 1. An agent a is a discrete entity aiming to classify a patient with respect to a set of classes. An agent a has its own belief base $B_{a}$ and a set of goals $G_{a}$.

In this paper, we will often appoint our agent as a dialogue agent. However, we would like to stress again that, here, the word dialogue means the whole process of interaction between the agent and the environment (practitioners, sensors, services, etc.), it is not limited to a conversation between the agent and the human actor.

Definition 2. The agent belief base $B_{a}$ contains the collection of the truth values (true, false, or unknown) associated with each information unit $s$, as well as a set of rules $R_{a}$ supporting the patient classification task by stating which information units are required to achieve each goal.

$B_{a}$ is updated after the arrival of a new piece of information that is relevant for changing the truth value of an information unit $s$ contained in $B_{a}$.

Definition 3. An information unit s (called hereafter "slot") is a relevant information, increasing the knowledge of the belief base $B_{a}$ necessary for achieving a goal $g$.

We consider different types of information: (i) information acquired directly by asking the patient (slots of type $S_{a}$ ), (ii) information acquired directly through sensors (slots of type $S_{b}$ ), (iii) information acquired from the patient's electronic file (slots of type $S_{c}$ ) (iv) information acquired from the history of data provided by the patient (slots of type $S_{d}$ ) and (v) information acquired through external services (slots of type $S_{e}$ ). The types of information required correspond to the ones presented in the guidelines.

The default value of a slot $s$ is unknown if no information concerning it is acquired. During the information acquisition process, the value of a slot s can change to true or false depending on the input.

Definition 4. A goal $g \in G_{a}$ is a class for which an agent a has to know if a patient is classified with it or not. The classification task is performed by applying the rules defined in $R_{a}$.

A goal can be characterized thanks to two values: (i) a confidence degree $C(g)$ representing the amount of true information (i.e., the truth value of a slot $s$ is true) that the agent $a$ collected about the goal $g$, and (ii) $M(g)$ representing the amount of information that the agent $a$ still have to collect for having a complete knowledge concerning 
the goal $g$, i.e., missing information. Let $\tau$ be a threshold given by an expert. A goal $g$ can be considered achieved (the patient may be considered as belonging or not to the class) if: (i) $C(g) \geq \tau$ or if (ii) $C(g)+M(g)<\tau$. The former means that the information collected by the agent $a$ is enough for classifying the patient as belonging to the class $g$. The latter means that even if all missing information would be set to true, the value of $C(g)$ will not pass the threshold $\tau$. Hence, the agent $a$ can classify the patient as not belonging to the class $g$. In both cases, the agent $a$ achieves the goal. This is one of the peculiarities of the healthcare domain, since a physician should be supported by intelligent agents able to both detect and exclude undesired situations.

Another aspect is the possibility of giving an importance degree to each goal $g$, hereafter called "priority".

Definition 5. The priority o of a goal $g$ is a real value in the interval $[0,1]$ representing the importance degree that the goal $g$ has within the belief base $B_{a}$. The set of all priority values is given by $O_{G_{a}}$

Definition 6. A rule $r \in R_{a}$ allows to classify a patient into a class $g$ based on the information collected by an agent $a$. A rule can be represented as follows: $s_{1} \oplus s_{2} \oplus$ $\ldots \oplus s_{n_{g}} \Rightarrow g$, where $s_{i}$ represents the ith slot related to $g$ and $n_{g}$ is the number of slots related to $g$.

Definition 7. The premise set $P(g)$ is the set of all the slots that help classifying goal $g$, i.e., $P(g)=\{s \mid s \in l h s(g)\}$, where "lhs" is the left hand side of a rule $r \in R_{a}$.

Definition 8. Let $g_{j}$ be a goal and $s_{i}$ be a slot. The association between $g_{j}$ and $s_{i}$ is represented through a real value $w_{i j} \in[0,1]$, called "weight". The weight represents how relevant the information contained in the slot $s_{i}$ is for achieving the goal $g_{j}$. We will also note the assotiation between goal $g$ with Slot $s$ as $w(s, g)$.

The same slot can appear in more than one rule $r$ since some information units can be shared by more than one goal. Each value $w_{i j}$ is directly provided by domain experts (guidelines) or inferred from background knowledge and its computation is out of scope of this paper. The only constraint is that, given the $j$ th goal, it holds the hypothesis $\sum_{i=0}^{n_{g_{j}}} w_{i j}=1$, where $n_{g_{j}}$ is the number of slots associated with the goal $g_{j}$. Even if this constraint holds, these values are not probabilities. The rationales behind this constraint are the following. First, we require that if all the slots associated with a specific goal are set to true, the agent is "sure" that the patient will be considered as belonging to the risky situation specified by the goal. Second, the classification of a patient with respect to a goal is performed by comparing the confidence that an agent has with respect to the possibility that a patient belongs to that goal, with a threshold $\tau$. Hence, in order to perform this comparison fairly, the numerical boundaries of the confidence has to be the same for all goals.

We introduce in Section 3 a working example that will be used throughout the paper and that will show how these elements can be instantiated into a real-world case.

\section{Working Example}

In this section, we present an illustrative example to facilitate understanding of the proposed framework and to show how the framework can be instantiated in a real-world 
scenario. In this example, whose aim is to help the reader understand our framework, we only consider the slots of type $S_{a}$, i.e. the slots concerning information obtained from the patient during a consultation with a practitioner.

Let us define a scenario in which, through dialogue, an agent aims to obtain the symptoms experienced by a patient in order to diagnose the possibility that the patient is in one or more risky situations which can occur in case of the chronic disease $\alpha$.

Here, we assume to have a finite set $G_{a}=\left\{g_{1}, g_{2}, g_{3}, g_{4}\right\}$ of goals expressing risky situations related to $\alpha$ and a finite set $S=\left\{s_{1}, \ldots, s_{d}\right\}$ of symptoms related to the different risky situations; $d$ corresponds to the number of slots (pieces of information) related to the different risky situations. We also have the assumptions that (i) each symptom $s$ (represented by a slot) can be a premise for more than one risky situation and (ii) the truth value of each slot $s$ can be 0 (i.e., the symptom has not been experienced) or 1 (i.e., the symptom has been experienced). For the continuous values, like "fever measures", etc., 1 and 0 means respectively that the value exceeds or is below the threshold according to the guidelines.

Table 1 shows the list of risky situations related to the chronic disease $\alpha$, the list of symptoms, and the weight values $w$ represent the associations between symptoms and the risky situation. These information compose the belief base $B_{a}$ that an agent $a$ has during the interaction with a patient. The aim of the agent is to achieve all the set of goals $G_{a}$ by classify the patient with respect to each risky situation, i.e., to know if the patient is in one or more risky situations or not.

Table 1: Chronic Disease $\alpha /$ Symptoms Domain Knowledge/Weights

\begin{tabular}{|c|c|c|c|c|c|c|c|c|c|c|c|}
\hline \multicolumn{10}{|c|}{ Risky Situations } & \multicolumn{10}{|c|}{} \\
\hline
\end{tabular}

The classification task is performed by applying the rules $R_{a}$ that allow to classify the patient with respect to each risky situation.

$$
\begin{array}{r}
s_{1} \oplus s_{2} \oplus s_{3} \oplus s_{4} \Rightarrow g_{1} \\
s_{1} \oplus s_{4} \oplus s_{5} \oplus s_{6} \oplus s_{7} \Rightarrow g_{2} \\
s_{1} \oplus s_{4} \oplus s_{6} \oplus s_{8} \oplus s_{9} \Rightarrow g_{3} \\
s_{4} \oplus s_{8} \oplus s_{10} \oplus s_{11} \oplus s_{12} \Rightarrow g_{4}
\end{array}
$$

Finally, we consider that the domain knowledge includes also information concerning entailments about co-occurrence of symptoms, for example a patient with high fever also has "just" fever. This knowledge is also provided by the experts. In our example, we assume to have the following three entailments:

$$
s_{6} \rightarrow s_{1} ; s_{1} \rightarrow s_{9} ; s_{10} \wedge s_{11} \rightarrow s_{4}
$$


We rely on this example in Section 4 when our framework is presented.

\section{Proposed Framework}

We propose a new agent-based framework for supporting the management of risky situations in the case of chronic diseases, thanks to (i) a slot filling interaction that is conducted with a patient (we will name such slots $S_{a}$ ), (ii) information acquired directly through sensors (we will name such slots $S_{b}$ ), (iii) information acquired from the patient's electronic file (we will name such slots $S_{c}$ ), (iv) information acquired from the history of data provided by the patient (we will name such slots $S_{d}$ ) (v) information acquired through external services (we will name such slots $S_{e}$ ). Not all the slots must be filled, i.e., depending on the risky situation, each set of slots can be instantiated with the information needed or not.

It is important to highlight that both, the conversion of a dialogue action into a natural language utterance and the natural language processing of the answer given by the patient are out of scope of this work.

\subsection{The Proposed Extension}

To address the challenge of effectively selecting the next dialogue action that most contributes for the classification (risky or not), we extend the framework proposed in [13] that models information usefulness for goal-driven agents. The rationales behind this choice are the following: (i) here, the main aim of the dialogue agent is to understand which is (or which are) the class to which the patient belongs to according to the values given to the slots (through the answers that he/she gives to the questions); (ii) such values can be viewed as the components in the left-hand side (lhs) of a rule, allowing to associate possible slots (e.g., symptoms) to a particular class (e.g., disease); and (iii) the dialogue agent aims to mimic the reasoning task of a physician, who can be considered as a cognitive agent, i.e., someone with a background knowledge (beliefs) about the patient and about other subjects (e.g., the possible relations or the probability of co-occurrence between the symptoms, etc.).

We consider a propositional language $L$ of which a subset, $L_{G}$, is the language used to represent the rules associated with the goals. We will consider a dialogue agent which is aware of both all the beliefs/knowledge (in addition to information concerning entailments, this includes the pieces of information obtained thanks to Slots of types $S_{b}, S_{c}, S_{d}$ and $S_{e}$ ), and the goals of the physician, as well as the patient's answers (slots of type $S_{a}$ ). The dialogue agent $a$ has a goal set $G_{a}$ (classes or risky situations) which corresponds then to the physician goals from the language of possible goals $L_{G}$. For example, in the context of managing chronic diseases, the goal of the agent is to understand from the different symptoms $\left(s_{1}, s_{2}, \ldots\right)$, if the patient is in a risky situations or not. We would like to notice that, unlike in [13] where the goal achievement is binary, here we consider a gradual definition to compute the extent to which a given situation is considered as risky and we consider a threshold $(\tau)$ which allows us to decide if the result is positive (the patient is in a risky situation) or not (the patient is not in a risky situation). 
Moreover, the dialogue agent $a$ has a belief base $B_{a}$ composed of two subsets $B_{a}^{m}$ and $B_{a}^{g} . B_{a}^{m}$ is the set of formulas from $L \backslash L_{G}$ which represents $a$ 's beliefs about the slot-values, e.g., the patient has pruritus $\left(s_{2}\right)$, the patient has fever $\left(s_{4}\right)$, nausea $\left(s_{6}\right)$, incontinence $\left(s_{7}\right)$, etc.. $B_{a}^{m}$ may also contain other physician background knowledge like the co-occurrence between the slots, e.g., Pain Abdominal implies Pain $\left(s_{1} \rightarrow\right.$ $s_{9}$ ). This means that, if Pain Abdominal is reported during the dialogue as one of the experienced symptoms by the patient, $B_{a}$ is automatically updated with both the values concerning the slots Pain Abdominal and Pain. $B_{a}^{g}$ instead, contains as many rules of the form $s_{1} \oplus s_{2} \oplus \ldots \oplus s_{n_{g}} \Rightarrow g$ (as stated in Def.6), where each $s_{i}$ is a literal of $L \backslash L_{G}$, representing a slot that influences the achievement of goal $g$, as there are $g \in G_{a}$. Such rules represent the beliefs of $a$ about which information is needed to determine a class, achieving then a given goal. These pieces of information are indeed the slot-values of the different types presented at the begining of this section. For example, to understand if the patient risks an Acute liver failure $\left(g_{1}\right)$, the physician needs to know if the patient has Pain Abdominal $\left(s_{1}\right)$, Pruritus $\left(s_{2}\right)$, Distress respiratory $\left(s_{3}\right)$, and Fever $\left(s_{4}\right)$, i.e., $s_{1} \oplus s_{2} \oplus s_{3} \oplus s_{4} \Rightarrow g$. The set of the elements in the left-hand-side of this rule is the premise of $g$ (Def.7), i.e., $P(g)=\left\{s_{1}, \ldots, s_{4}\right\}$.

Let us suppose that the agent acquires more and more information about the patient's actual state, approaching the classification into one or more risky situations. The units of information that are still missing can be represented as follows.

Definition 9 (Missing Information). Let a be a dialogue agent with its belief base $B_{a}$ and its goal set $G_{a}$. Let $g \in G_{a}$ be such that $B_{a} \not \neq g{ }^{4}$ The missing information for goal $g$, Missing $\left(B_{a}, g\right)$, is defined as follows:

$$
M i \operatorname{sing}\left(B_{a}, g\right)=\left\{l: l \in P(g) \text { and } B_{a} \not \models l\right\}
$$

$\operatorname{Missing}\left(B_{a}, g\right)$ is the set of all the slots in the premise of $g$ which cannot be deduced from $B_{a}$ (i.e., which are not yet believed by the agent and therefore the dialogue agent should acquire them).

Remark 1. In the particular case in which $B_{a}^{m}=\emptyset, \operatorname{Missing}\left(B_{a}, g\right)=P(g)$, i.e., the missing piece of information to achieve $g$ is $P(g)$.

Because different goals can have missing information in common, we need to introduce the notion of multiset of missing information.

Definition 10 (Multiset of missing information). Let a be a dialogue agent whose belief base is $B_{a}$ and whose goal set is $G_{a}$. The multiset ${ }^{5}$ of missing information to achieve the goals in $G_{a}$ is:

$$
\operatorname{Missing}\left(B_{a}, G_{a}\right)=\bigcup_{k=1}^{\left|G_{a}\right|} \operatorname{Missing}\left(B_{a}, g_{k}\right)
$$

with $\bigcup$ representing the union on multisets, || representing the cardinality of a set.

\footnotetext{
${ }^{4}$ In propositional logic, $\phi=\psi$ means that $\psi$ is a logical consequence of $\phi$. Here, it means that we can classify the patient as having disease $g$ from what we already know/believe $\left(B_{a} \models g\right.$, see Defi. 15.

${ }^{5}$ Reminder: a multiset is a set whose elements can have several occurrences, such as $\{p, q, p\}$.
} 
Let us consider the following definitions:

Definition 11. Let $G(s)$ be the set of goals related to slot s. We define $G(s)=\{g \in$ $\left.G_{a} \mid s \in P(g)\right\}$.

Definition 12. Let $W(g)$ be the set of all the weights related to the symptoms associated to goal $g . W(g)=\{w(s, g) \mid s \in P(g)\}$.

We can now define the overall importance of requiring an answer concerning slot $s$, $N_{1}(s)$, (which represents the extent to which $s$ would help getting closer to a classification), with respect to all the goals $g \in G_{a}$ as follows:

$$
N_{1}(s)=\left\{\begin{array}{l}
\sum_{g \in G(s)} w(s, g)+\sum_{s^{\prime}\left|B_{a} \cup s\right|=s^{\prime} \wedge B_{a} \not \models s^{\prime}} w\left(s^{\prime}, g\right) \text { if } s=\text { true, } \\
\sum_{g \in G(s)} w(s, g)+\sum_{s^{\prime}\left|B_{a} \cup s^{\prime}\right|=s \wedge B_{a} \not \models s} w\left(s^{\prime}, g\right) \text { if } s=\text { false. }
\end{array}\right\}
$$

where $w\left(s^{\prime}, g\right)=0$ if $s^{\prime} \notin P(g)$ and $s$ comes from the multiset of missing information (Def. 10). We can notice that $N_{1}(s)$ is the gradual definition (and therefore an extension) of the $N_{1}$ component proposed in [13].

We can also compute the overall weight, $N_{2}(s)$, that concerns the slots which are still missing after receiving the value of slot $s$ as follows:

$$
N_{2}(s)=\left(\sum_{g \in G_{a}} \sum_{k \in W(g)} k\right)-N_{1}(s) .
$$

We can notice that our definition of $N_{2}(s)$ is a generalization (a gradual counterpart) of the one proposed in [13].

To characterize a goal as being achieved or not, we need to know the amount of already known information about the slots related to it and the amount of information which is still missing.

Definition 13. The amount of information that the agent a collected about the goal $g$, is given by the following two measures:

$$
\mathcal{C}(g)=\sum_{\left(s \mid B_{a} \models s\right) \wedge(s \in P(g))} w(s, g)
$$

representing the information slot set to true, and:

$$
\mathcal{C}(\neg g)=\sum_{\left(s \mid B_{a} \models \neg s\right) \wedge(s \in P(g))} w(s, g)
$$

representing the information slot set to false.

Definition 14. The amount of information that the agent a still has to collect for having a complete knowledge concerning goal $g, \mathcal{M}(g)$ is given by:

$$
\mathcal{M}(g)=\sum_{s^{\prime} \in M i \sin g\left(B_{a}, g\right)} w\left(s^{\prime}, g\right)
$$

Let $\tau$ be a threshold which allows to characterize a goal as being achieved or not, according to the guidelines. 
Definition 15. A goal $g$ is achieved, i.e., we can assert that the patient is in a risky situation, noted $B_{a} \models g$, if and only if:

$-\mathcal{C}(g) \geq \tau:$ the amount of information already available is sufficient to make a positive diagnosis, i.e., the patient's life is in danger, $\mathrm{Or}$

$-\mathcal{C}(g)+\mathcal{M}(g)<\tau$ : the amount of information already available is sufficient to make a negative diagnosis, i.e., the patient's life is not in danger.

The value of $\tau$ obviously influences the capability of an agent concerning the achievement of goals. High values of $\tau$ reduces the possibility of classifying a patient as belonging to a specific goal, but increases the possibility that a patient would not belong to any goal. The opposite occurs for low values of $\tau$.

Remark 2. We can notice that if there are not missing slots, i.e., $\mathcal{M}(g)=0$, we have: $\mathcal{C}(g)+\mathcal{C}(\neg g)=1$.

This remark shows how the total amount of information is preserved when all slots are filled.

Definition 16. The set of goals that a slot s allows the dialogue agent to achieve is:

$$
E(s)=\left\{g \in G_{a}\left|B_{a} \cup s\right|=g \wedge B_{a} \not \models g\right\} .
$$

\subsection{Next Question Selection}

This work provides a function that measures the usefulness of a (not-yet-filled) slot 6 By comparing the usefulness value of the different slots, it is possible to select the best candidate question/test/check for the next information acquisition, i.e., the one that allows us to achieve/get closer to a classification.

The usefulness measure takes into account several factors:

- Class' priority: a priority can be set to each class. Therefore, whenever priority values are available, slots related to classes with higher priority have their usefulness value increased. Our working example does not present priorities among the classes, therefore the neutral value 1.0 is assigned.

- Information filled by the slot: some slots are capable of filling more information than others, since they are related to more than one class or the domain knowledge reports some co-occurrence or entailement.

- Slot's weight: as previously stated, according to the domain, not all pieces of information (slots) have the same relevance with respect to a class. For example, a physician may inform that abdominal pain $\left(s_{1}\right)$ is more relevant than fever $\left(s_{4}\right)$ for determining that the patient has Acute liver failure $\left(g_{1}\right)$ and, therefore, the latter would present a higher usefulness value to this class. The usefulness measure also takes into account that a slot can be present in different classes with different weights.

\footnotetext{
${ }^{6}$ Of course, here we are interested in knowing the utility of the slots which are not a logical consequence of the belief base $B_{a}$, i.e., which cannot be deduced from previous beliefs/knowledge.
} 
Due to the different requirements of health domains, when we compute the usefulness value of a slot $s$, we need to consider what happens in both cases, i.e. if the slots $s$ is set to 1 (the symptom occurred) or to 0 (the symptom did not occur). The resulting equation for computing the usefulness of a slot $s$ is the following:

$$
\begin{aligned}
U(s)=\left[\left(|E(s)|+\frac{N_{1}(s)}{N_{1}(s)+N_{2}(s)}\right)^{s=0}+\right. & \\
& \left.\left(|E(s)|+\frac{N_{1}(s)}{N_{1}(s)+N_{2}(s)}\right)^{s=1}\right] \frac{O_{G_{a}}^{s}}{G_{a}^{s}}
\end{aligned}
$$

where:

- $|E(s)|$ is the number of goals that are satisfied thanks to the information about slot $s$, i.e., the number of classes (risky situations) for which we can conclude if the patient belongs to them or not after checking the slot $s$;

- $N_{1}(s)$ and $N_{2}(s)$ are computed by taking into account the weights associated with $s$ in all goals;

- $O_{G_{a}}^{s}$ is the sum of the priorities associated with the goals having the slot $s$ in their premise.

- $\left|G_{a}^{s}\right|$ is the number of goals having the slot $s$ in their premise.

The coefficient $\frac{O_{G_{a}}^{s}}{\left|G_{a}^{s}\right|}$ allows to increase the usefulness value of slots that are premise of more important goals.

The proposed approach follows the principle of coverage, i.e., it aims to explore the domain as much as possible in order to investigate different possibilities that may lead to a classification. As defined in the proposed function, this is achieved by increasing the usefulness value of slots that, besides enabling the achievement of as many goals as possible, also cover more information from a single action for information acquisition (question/test/check), such those that are common to more than one cluster or that cooccur. For example, obtaining the value for the symptom Abdominal pain $\left(s_{1}\right)$ will cover not only one, but some risky situations $\left(g_{1}, g_{2}, g_{3}\right)$ and also the symptom Pain $\left(s_{9}\right)$, due to the entailment stated in Section 3 , therefore, its usefulness value is increased (because $N_{1}(s)$ increases). The main advantage of this approach is to empower the cautiousness of the practitioner within sensitive scenarios. As a disadvantage, this approach may be slow to reach the classification when $G_{a}$ is a large set.

\section{The Framework in Action}

We discuss here the application of the proposed framework to the working example described in Section 3 For demonstration purposes, we set the value of $\tau$ to 0.75 . Let us suppose to have a dialogue started by a patient in which he/she reported a general illness and the fever and nausea symptoms.

Step 1: the agent acquires these two information and set the truth values for the slots $s_{4}$ and $s_{6}$ to 1 .

Step 2: by analyzing the co-occurrence knowledge, the agent sets also the slots $s_{1}$ and $s_{9}$ to 1 from the application of both the $s_{6} \rightarrow s_{1}$ and $s_{1} \rightarrow s_{9}$ entailments. $C_{G_{a}}$ values change in: $C_{g_{1}}=0.52, C_{g_{2}}=0.61, C_{g_{3}}=0.58, C_{g_{4}}=0.13$. 
Step 3: the agent computes $U(s)$ for the remaining slots. The three most useful slots resulted: $U\left(s_{8}\right)=4.05, U\left(s_{2}\right)=2.19, U\left(s_{5}\right)=2.18$.

Step 4: the agent asks for the symptom associated with $s_{8}$ (i.e. Shortness of breath) and the patient replies that he/she does not experiences this. The slot $s_{8}$ is set to 0 , and the different values of $\mathcal{C}(g)$ remain unchanged. However, by checking the values of $\mathcal{C}(g)+\mathcal{M}(g)$ for the goals $g_{3}$ and $g_{4}$, we can observe the values of 0.58 and 0.65 respectively. This means that the agent can already exclude two possible risky situations since even if all missing information would be set to 1 , both $\mathcal{C}\left(g_{3}\right)$ and $\mathcal{C}\left(g_{4}\right)$ will be lower than $\tau$.

Step 5: the agent computes $U(s)$ for the remaining slots. The two most useful slots resulted: $U\left(s_{2}\right)=2.40, U\left(s_{5}\right)=2.37$, while the others have values lower than 0.5 .

Step 6: the agent asks for the symptom associated with $s_{2}$ (i.e. Pruritus) and the patient replies that he/she does experiences this. The slot $s_{2}$ is set to 1 and the different values change in: $\mathcal{C}\left(g_{1}\right)=0.80, \mathcal{C}\left(g_{2}\right)=0.61, \mathcal{C}\left(g_{3}\right)=0.58$, and $\mathcal{C}\left(g_{4}\right)=0.13$. This answer allows the agent to conclude that the patient is in the risky situation $g_{1}$.

Here, based on the decision policy applied by the domain expert, we can obtain different behaviors. For example, the adoption of a cautious approach can bring the agent to perform other steps as shown below.

Step 7: the agent computes $U(s)$ for the remaining slots. The most useful slot resulted $U\left(s_{5}\right)=2.47$, while the others continue to have values lower than 0.5 .

Step 8: the agent asks for the symptom associated with $s_{5}$ (i.e. Early awakening) and the patient replies that he/she does not experiences this. The slot $s_{5}$ is set to 0 , and the different values of $\mathcal{C}(g)$ remain unchanged. Hence, the goal (class) $g_{1}$ remains the only goal which the patient belongs to. Indeed, by checking the values of $\mathcal{C}(g)+\mathcal{M}(g)$ for the different goals, we can observe a value of 0.74 for the goals $g_{2}$. This means that the agent excludes also this risky situation since it becomes impossible for $\mathcal{C}\left(g_{2}\right)$ to reach the threshold $\tau$.

Differently, in case of a positive answer for the slot $s_{5}$, the different values of $\mathcal{C}(g)$ would change in: $\mathcal{C}\left(g_{1}\right)=0.80, \mathcal{C}\left(g_{2}\right)=0.87, \mathcal{C}\left(g_{3}\right)=0.58, \mathcal{C}\left(g_{4}\right)=0.13$. Hence, the agent would conclude that the patient is in two possible risky situations. Even if this situation somehow introduces uncertainty in the analysis of patient's conditions because there are more than one risky situation, it is a duty of the domain expert to decide which actions to take. This practice is commonly accepted within the healthcare domain as intelligent systems aim to support domain experts and not to replace them.

\section{Evaluation}

The previous section discusses a small running example showing in practice which are the execution steps performed by using the proposed framework. The evaluation of the proposed framework has been performed on two real-world scenarios concerning the management of risky situations in two chronic diseases, namely type-2 diabetes (this scenario is hereafter referred as $S M I$ ) and of asthma (this scenario is hereafter referred as $S M 2)$.

SMI requires the monitoring of food intake and physical activities performed by a patient during specific time-spans with respect to a set of guidelines. In this scenario, we encoded the guidelines provided by the American Diabetes Association (ADA) 7

\footnotetext{
${ }^{7}$ https://www.diabetes.org/
} 
The encoding of such guidelines corresponded to a set of 87 goals and 174 information slots grouped as follows:

- 79 slots of type $S_{a}$ corresponding to food categories that are relevant for the ADA guidelines and that a patient can explicitly provide;

- 4 slots of type $S_{b}$ corresponding to values acquired from the glucometer before and after a meal or a physical activity session;

- 12 slots of type $S_{c}$ corresponding to information acquired from personal health records of patients concerning past hospitalizations, upsets, etc.;

- 79 slots of type $S_{d}$ corresponding to the last-week intake concerning food categories that are relevant for the ADA guidelines and that are acquired from an external knowledge repository.

While, $S M 2$ requires the monitoring of respiratory issues from two perspectives: (i) by processing the symptoms provided by a patient; and, (ii) by providing recommendations based on the analysis of patient profile and data acquired from external services. In this scenario, we encoded the guidelines provided by the American Lung Association (ALA) ${ }^{8}$. The encoding of such guidelines corresponded to a set of 7 goals and 69 information slots grouped as follows:

- 22 slots of type $S_{a}$ corresponding to the symptoms that are relevant for the ALA guidelines and that a patient can explicitly report;

- 11 slots of type $S_{b}$ corresponding to the type of parameters that are acquired from inhalers and other medication devices;

- 8 slots of type $S_{c}$ corresponding to information acquired from personal health records of patients concerning past hospitalizations, upsets, etc.;

- 16 slots of type $S_{d}$ corresponding to patient's habits that are relevant for the ALA guidelines and that are acquired from an external knowledge repository (e.g. if a patient uses to go running on a specific day of the week);

- 12 slots of type $S_{e}$ corresponding to information that are relevant for the ALA guidelines and that are acquired from external services (e.g. weather forecast, pollen forecast, etc.).

We validated the effectiveness of the proposed framework with respect to three baselines:

- Baseline 1 (BS1): at each iteration, the selected slot is the most popular one. This means that the agent will check in its knowledge which is the information slot valued as "unknown" and having the highest number of occurrences.

- Baseline 2 (BS2): at each iteration, the selected slot is the one with the highest overall weight within the knowledge base. The overall weight of a slot $s$ is computed by summing all the weights $w(s, g)$ associated with the slot $s$ in the entire set of goals $G_{a}$.

- Baseline 3 (BS3): this baseline is a variation of BS1 where at each iteration, the selected slot is the one having the highest normalized frequency. The normalized frequency of a slot $s$ is computed by dividing the number of occurrences of $s$ by the number of slots valued as "unknown" within the knowledge base.

\footnotetext{
${ }^{8}$ https://www.lung.org/
} 
We analyzed three metrics: precision, recall, and the average number of steps required by the agent for classifying the patient. In our setting, the algorithm stopped when for at least one goal the expression $\mathcal{C}(g) \geq \tau$ is true. Precision and recall are computed by checking if the set of goals for which the expression above is true contains the correct one. While, the average number of steps is computed by considering the number of information request performed by the agent before reaching a conclusion.

The evaluation has been performed by simulating the interactions between the agent and a set of patients. The simulations have been though in order to cover all goals defined in each scenario. Since in this work we did not take into account the analysis of how the value of $\tau$ can be set, we run a simulation for each possible value of $\tau$ within the interval $[0.00,1.00]$ with an incremental step of 0.01 between two consecutive simulations. Finally, each simulation starts with a fixed number of slots already set. These slots represent the information provided by the patient when she starts the interaction with the system. For each value of $\tau$, we performed three simulations with 1,2 , or 3 slots set on startup, respectively. This parameter made it possible to observe how the effectiveness of our approach changes according to the amount of information provided at startup.

Figures 1, 2, and 3 show the results observed on SM1, while Figures 4, 5 , and 6 show the results observed for SM2. Within all figures, the x-axis reports the different values of $\tau$, while the y-axis reports the precision and recall observed at each step or the average number of steps.

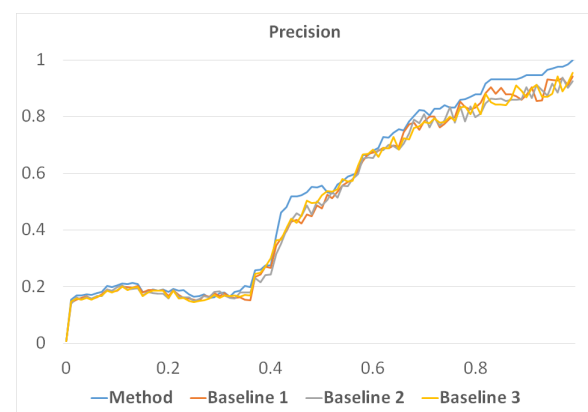

Fig. 1: Diabetes use case: graph of the Precision metric observed for all values of the threshold $\tau$ within the interval $[0.0,1.0]$.

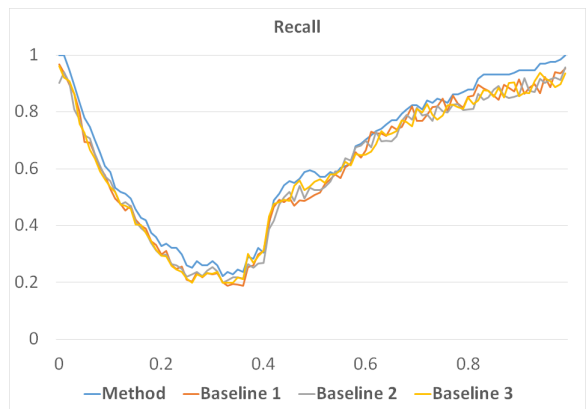

Fig. 2: Diabetes use case: graph of the Precision metric observed for all values of the threshold $\tau$ within the interval $[0.0,1.0]$.

The analysis of the obtained results highlighted four main points of interest.

By observing the graphs shown in Figures 3 and 6 , we can notice how at a certain point the number of steps decreases. This behavior is quite strange by considering that by increasing the value of $\tau$ the expected number of steps needed for passing the threshold should increase as well. A more deep analysis revealed that by adopting our framework for selecting the next slot to fill, the agent increases the capability of detecting unreachable goals. Hence, it is possible for the agent to reduce the number of steps for classifying the patient with respect to the correct goal. 


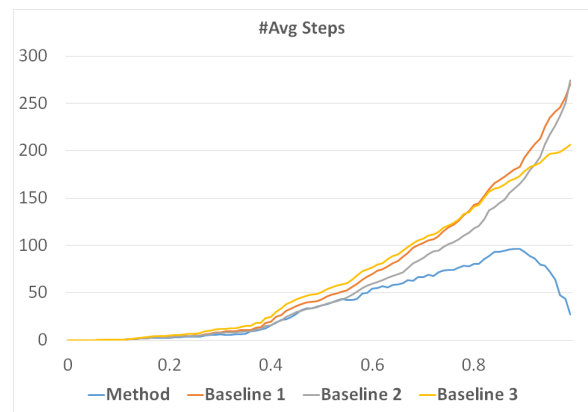

Fig. 3: Diabetes use case: graph of the number of steps required by the agent to reach a conclusion observed for all values of the threshold $\tau$ within the interval $[0.0,1.0]$.

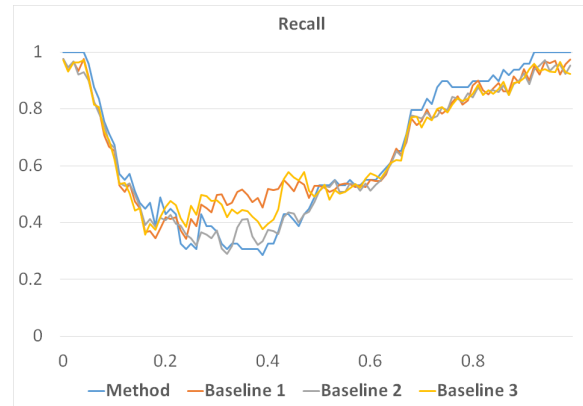

Fig. 5: Asthma use case: graph of the Precision metric observed for all values of the threshold $\tau$ within the interval $[0.0,1.0]$.

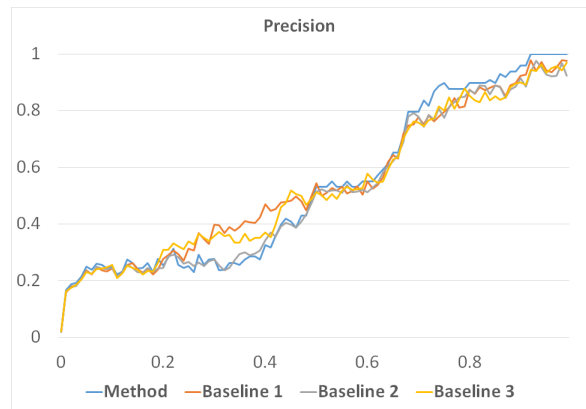

Fig. 4: Asthma use case: graph of the Precision metric observed for all values of the threshold $\tau$ within the interval $[0.0,1.0]$.

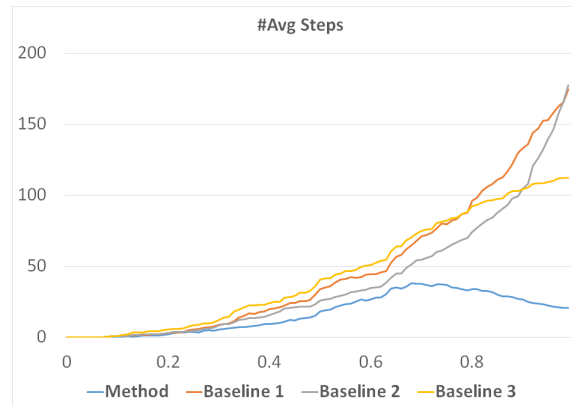

Fig. 6: Asthma use case: graph of the number of steps required by the agent to reach a conclusion observed for all values of the threshold $\tau$ within the interval $[0.0,1.0]$.

Together with the reduction of the number of steps required by the agent for reaching a conclusion, we can observe also an improvement of the overall effectiveness of our framework. Indeed, for high values of $\tau$, corresponding to the need of having a high confidence about the conclusion provided by the agent, the proposed framework outperforms all the baselines for both the precision and recall values.

By focusing on the recall graph, there are a couple of aspects that attracted our attention: the high recall observed for low $\tau$ values and the subsequent drop. The former is justified by the fact that the patient starts the interaction by providing some information, i.e. some slots are filled on startup. Thus, for low values of $\tau$, the possibility that the correct goal is contained within the set of goals passed the threshold is high. On the contrary, the precision values are low since, especially if the filled slots are very popular, many goals can be satisfied by considering only the information provided on 
startup. The latter is given by considering that, medium values of $\tau$ means that the agent is in the middle of acquiring information and it seems that in many cases there are goals, then resulted to be the wrong ones, that are satisfied with this partial knowledge. Future work will consider to investigate more on this behavior.

Finally, by comparing the two scenarios, even if the proposed framework outperforms the baselines in both of them, it resulted to be more effective in the most complex one. A possible reason is that within scenarios having a low number of information slots, strategies relies on frequencies can be more effective, especially for lower values of $\tau$. However, it is important to remark two aspects. First, real-world scenarios within the healthcare domain considers precision values in the range of $0.4-0.6$ not acceptable by domain experts. Hence, the possibility of adopting low values of $\tau$ in practice is very low. Second, the proposed framework demonstrated to be more effective with respect to the baselines by exploiting a dramatically lower number of interactions. The latter is a very important aspect within the healthcare domain since it allows to understand which is the current healthy status of a patient and, at the same time, to reduce the effort for providing the knowledge required for reaching a conclusion.

\section{Final Remarks}

We presented a goal-based framework to support the management and control of risk situations for chronic diseases. We discussed the concept of information usefulness in a health dialogue and we formalized the metrics that allow to compute the usefulness value of a missing slot. A running example about diagnosis has been used for showing how the framework can be instantiated.

As previously mentioned, the proposed usefulness measure has as one of its main benefits the generation of a "short" information acquisition process due to the careful selection of the slot that most contributes with usefull information. As further benefits, this approach avoids the need of large historical databases and the costs of supporting costly information acquisition to obtain a reliable output.

By proposing this approach, we do not claim to cover the state transition of the information acquisition process, which is also a role of the practitioner. Instead, we intend to contribute as a heuristic measure that covers the needs of the health domain to support the choice of the next dialogue action. This heuristic can be applied together with some of the strategies, for example, those currently used for dialogue management, such as automated planning [14] or finite state machines [15], which can also benefit from the content of the belief base $B_{a}$ to determine the dialogue state.

To increase the elicitation of information, some other strategies can also be integrated in a dialogue system, such as the use of a mixed-initiative design associated to the manipulation of open answers, which would allow the spontaneous input of information by the patient.

This work opens several research directions to be accomplished in the future. First, in this work we considered binary values for setting the slots. The use of fuzzy sets, allowing to confirm a slot to a certain degree, would be an interesting extension of the proposed framework. Second, we will focus on designing an evaluation protocol for validating agents instantiating our framework. Finally, we plan to integrate and deploy our framework into existing architectures in order to start its application into real-world scenarios. 


\section{Acknowledgement}

Célia da Costa Pereira acknowledges support of the PEPS AIRINFO project funded by the CNRS. This work has been carried out during her visit at Process \& Data Intelligence research unit at Fondazione Bruno Kessler of Trento.

\section{References}

1. Dennis, S., Zwar, N., Griffiths, R., Roland, M., Hasan, I., Powell Davies, G., Harris, M.: Chronic disease management in primary care: From evidence to policy. The Medical journal of Australia 188 (05 2008) S53-6

2. Johnson, P., Tu, S., Booth, N., Sugden, B., Purves, I.: Using scenarios in chronic disease management guidelines for primary care. Proceedings / AMIA ... Annual Symposium. AMIA Symposium (02 2000) 389-93

3. Hernández, D., Villarrubia, G., Barriuso, A.L., Lozano, Á., Revuelta, J., De Paz, J.F.: Multi agent application for chronic patients: Monitoring and detection of remote anomalous situations. In: Highlights of Practical Applications of Scalable Multi-Agent Systems. The PAAMS Collection, Cham, Springer International Publishing (2016) 27-36

4. Park, H.S., Cho, H., Kim, H.S.: Development of a multi-agent m-health application based on various protocols for chronic disease self-management. Journal of Medical Systems 40(1) (Nov 2015) 36

5. Kraus, S.: Intelligent agents for rehabilitation and care of disabled and chronic patients. In: AAAI, AAAI Press (2015) 4032-4036

6. Huygens, M.W.J., Swinkels, I.C.S., de Jong, J.D., Heijmans, M.J.W.M., Friele, R.D., van Schayck, O.C.P., de Witte, L.P.: Self-monitoring of health data by patients with a chronic disease: does disease controllability matter? BMC family practice 18(1) (March 2017) 40

7. Croatti, A., Montagna, S., Ricci, A., Gamberini, E., Albarello, V., Agnoletti, V.: BDI personal medical assistant agents: The case of trauma tracking and alerting. Artificial Intelligence in Medicine 96 (2019) 187-197

8. Abdel-Basset, M., Manogaran, G., Gamal, A., Chang, V.: A novel intelligent medical decision support model based on soft computing and iot. IEEE Internet Things J. 7(5) (2020) $4160-4170$

9. Moreira, M.W.L., Rodrigues, J.J.P.C., Korotaev, V., Al-Muhtadi, J., Kumar, N.: A comprehensive review on smart decision support systems for health care. IEEE Syst. J. 13(3) (2019) 3536-3545

10. Kamiali, A., Riao, D., Kert, S., Welzer, T., Zlatolas, L.N.: Multi-level medical knowledge formalization to support medical practice for chronic diseases. Data and Knowledge Engineering 119 (2019) $36-57$

11. Elwenspoek, M.M.C., Patel, R., Watson, J.C., Whiting, P.: Are guidelines for monitoring chronic disease in primary care evidence based? BMJ 365 (2019)

12. Manning, C.L.: Artificial intelligence could bring relevant guidelines into every consultation. BMJ 366 (2019)

13. Cholvy, L., da Costa Pereira, C.: Usefulness of information for goal achievement. In: PRIMA. Volume 11873 of Lecture Notes in Computer Science., Springer (2019) 123-137

14. Behnke, G., Nielsen, F., Schiller, M., Ponomaryov, D., Bercher, P., Glimm, B., Minker, W., Biundo, S.: To plan for the user is to plan with the user: Integrating user interaction into the planning process. In: Companion Technology. Springer (2017) 123-144

15. Lee, K., Lee, Y.S., Nam, Y.: A model of fsm-based planner and dialogue supporting system for emergency call services. The Journal of Supercomputing 74(9) (2018) 4603-4612 